Check for updates

Cite this: Phys. Chem. Chem. Phys., $2020,22,16630$

Received 27th April 2020,

Accepted 9th July 2020

DOI: $10.1039 / \mathrm{d} 0 \mathrm{cp} 02251 \mathrm{a}$

rsc.li/pccp

\title{
Biofouling affects the redox kinetics of outer and inner sphere probes on carbon surfaces drastically differently - implications to biosensing $\dagger$
}

\author{
Emilia Peltola, (D)*a Anja Aarva, ${ }^{a}$ Sami Sainio, ${ }^{\text {bc }}$ Joonas J. Heikkinen, (D) d \\ Niklas Wester, ${ }^{d}$ Ville Jokinen, (D) ${ }^{d}$ Jari Koskinen (D) ${ }^{d}$ and Tomi Laurila (D) ${ }^{a}$
}

\begin{abstract}
Biofouling imposes a significant threat for sensing probes used in vivo. Antifouling strategies commonly utilize a protective layer on top of the electrode but this may compromise performance of the electrode. Here, we investigated the effect of surface topography and chemistry on fouling without additional protective layers. We have utilized two different carbon materials; tetrahedral amorphous carbon (ta-C) and SU-8 based pyrolytic carbon (PyC) in their typical smooth thin film structure as well as with a nanopillar topography templated from black silicon. The near edge $X$-ray absorption fine structure (NEXAFS) spectrum revealed striking differences in chemical functionalities of the surfaces. PyC contained equal amounts of ketone, hydroxyl and ether/epoxide groups, while ta-C contained significant amounts of carbonyl groups. Overall, oxygen functionalities were significantly increased on nanograss surfaces compared to the flat counterparts. Neither bovine serum albumin (BSA) or fetal bovine serum (FBS) fouling caused major effects on electron transfer kinetics of outer sphere redox (OSR) probe $\left.\mathrm{Ru}_{(\mathrm{NH}}\right)_{6}{ }^{3+}$ on any of the materials. In contrast, negatively charged OSR probe $\mathrm{IrCl}_{6}^{2-}$ kinetics were clearly affected by fouling, possibly due to the electrostatic repulsion between redox species and the anionically-charged proteins adsorbed on the electrode and/or stronger interaction of the proteins and positively charged surface. The OSR probe kinetics were less affected by fouling on PyC, probably due to conformational changes of proteins on the surface. Dopamine (DA) was tested as an inner sphere redox (ISR) probe and as expected, the kinetics were heavily dependent on the material; PyC had very fast electron transfer kinetics, while ta- $C$ had sluggish kinetics. DA electron transfer kinetics were heavily affected on all surfaces by fouling ( $\Delta E_{\mathrm{p}}$ increase $30-451 \%$ ). The effect was stronger on PyC, possibly due to the more strongly adhered protein layer limiting the access of the probe to the inner sphere.
\end{abstract}

\section{Introduction}

Biofouling has been proposed as the main reason for sensor failure in vivo. ${ }^{1}$ Fouling leads to reduced analyte diffusion and perfusion to implanted sensors severely affecting the analytical characteristics of a sensor, such as sensitivity, temporal resolution and overall reliability. As the majority of the electrochemical performance research is done in buffer solutions, the reliable

\footnotetext{
${ }^{a}$ Department of Electrical Engineering and Automation, School of Electrical Engineering, Aalto University, Espoo, Finland. E-mail: emilia.peltola@aalto.fi

${ }^{b}$ Stanford Synchrotron Radiation Lightsource, SLAC National Accelerator Laboratory, Menlo Park, CA 94025, USA

${ }^{c}$ Microelectronics Research Unit, Faculty of Information Technology and Electrical Engineering, University of Oulu, Oulu, Finland

${ }^{d}$ Department of Chemistry and Materials Science, School of Chemical Technology, Aalto University, Espoo, Finland

$\dagger$ Electronic supplementary information (ESI) available. See DOI: 10.1039/ d0cp02251a
}

assessment of sensor performance is not unambiguous. For example, the detection limit for dopamine (DA) can deteriorate from $50 \mathrm{nM}$ measured in phosphate buffered saline (PBS) to $50 \mu \mathrm{M}$ in a biological environment. ${ }^{2}$

Biofouling literally means passive adsorption of proteins and lipids. According to a widely accepted assumption, proteins form a monolayer on the surfaces, ${ }^{3}$ but the type of the adsorbed proteins and their conformation can be controlled. To maintain electrode performance in biological environment, material designers have developed different antifouling strategies. This means prevention or reduction of protein adsorption. From an electrochemical point of view, the critical thing is that the analyte has access to the electrode. Therefore, having control over protein adsorption rather than the reduction of protein adsorption plays a pivotal role in using electrochemical sensors successfully in a biological environment. Most antifouling strategies exploit a protective layer or barrier on an electrode substrate to prevent the fouling agent from reaching the 
electrode surface. ${ }^{4}$ For example, noble metal electrodes cannot be used in direct electrochemical detection of biomolecules as they suffer from severe biofouling and have tendency to exhibit high background current and, furthermore, are easily attacked in physiological conditions by chloride ions. Instead, they are commonly coated with polymers or other materials. ${ }^{5,6}$ For the detection of many biological analytes, this is not as suitable strategy, because (1) such films give rise to an increased background electrode capacitance and impedance, and (2) act as a diffusion barrier and as a result, a decreased amount of the analyte reaches the electrode surface and the reaction kinetics are compromised. Consequently, the sensitivity and temporal resolution of the electrode are sacrificed.

In this study, we propose that it is possible to control protein fouling just by modifying the electrode material and we show how to achieve improved performance in biological environment. In general, surface roughness increases protein adsorption. This is partly due to the increase of electrochemically active surface area, but with certain proteins the saturation uptake increases beyond the accompanying increase in surface area. ${ }^{7}$ However, with black silicon (bSi) based structures, it has been observed that with increasing levels of protein adsorption occurring at the top of the nanopillars, an increased amount of nanopillar clustering was observed, as a result of the increased protein concentration at that location. In this case, the interpillar space was not filled with proteins, despite the interpillar space being filled with water. ${ }^{8}$

Here, we utilized two different carbon materials, tetrahedral amorphous carbon and SU-8 based pyrolytic carbon in their typical smooth thin film structure as well as bSi nanopillar based topography. Black silicon is a synthetic nanostructured material that contains high-aspect-ratio nano-protrusions, such as spikes or needles, on the Si surface produced via plasma etching. Black diamond and bSi have been investigated as antibacterial surfaces. ${ }^{9,10}$ The extensive $\mathrm{sp}^{3}$ structure of diamond together with the typically low surface polar functional groups results in a dramatic decrease in the adsorption of certain fouling agents. ${ }^{4}$ Furthermore, boron doped diamond has been found to be more resistant to biofouling than glassy carbon and surface oxides of the glassy carbon electrode seemed to reduce the impact of adsorption on catalytic sites. ${ }^{11}$ Overall, the polar and dispersion components of the surface energy may affect protein adsorption as much or even more than the overall hydrophobicity. ${ }^{12}$

In this paper, we focus on the effect of protein adsorption on electrochemical performance. We utilize two models for fouling: (1) a simple single protein in a buffer model and (2) a more complex media with several proteins that provides a more realistic insight into the functional role of adsorbed proteins. Examples of the effects of fouling on electrochemical performance under both conditions are presented.

\section{Materials and Methods}

\section{Surface preparation}

Highly boron doped $100 \mathrm{~mm}$ silicon wafer $(\mathrm{p}++,\langle 100\rangle$, Siegert Wafer) were used as the substrate for all of the samples. Four sample types, ta-C and $\mathrm{PyC}$ in their typical smooth thin film structure as well as in bSi based topography, referred as nanograss-ta-C and nanograss-PyC. Fig. 1 presents a scheme that summarizes the electrode fabrication.

The bSi masters were fabricated with maskless anisotropic silicon etching in Inductively Coupled Plasma Reactive Ion Etching (ICP-RIE, Plasmalab 100 - ICP 180, Oxford Instruments) equipment with a process described previously. ${ }^{13}$ No pretreatments were done for silicon wafers before etching. The etching recipe was: SF6 ow $40 \mathrm{sccm}, \mathrm{O} 2$ ow $18 \mathrm{sccm}$, pressure $10 \mathrm{mTorr}$, temperature $-110{ }^{\circ} \mathrm{C}$, RF-power $6 \mathrm{~W}$, ICP-power $1000 \mathrm{~W}$, etch time five (PyC master) to seven (ta-C master) minutes.

The $7 \mathrm{~nm}$ ta-C coating was deposited with pulsed filtered cathodic vacuum arc (p-FCVA). Flat counterparts were fabricated with $20 \mathrm{~nm}$ Ti adhesion layer, while no adhesion layer was utilized for nanograss-ta-C structures. In p-FCVA an electric arc is initiated between a graphite cathode and an anode body in high vacuum. The high current density of the cathodic arc creates plasma as well as particles. The particles are filtered out and the plasma directed to the substrate through a $45^{\circ}$ angle magnetic filter, where it forms an $\mathrm{sp}^{3}$ rich thin film. The deposition process is described in greater detail in ref. 14 . The samples were diced with Disco DAD3220 dicing saw into $10 \times 10 \mathrm{~mm}^{2}$ pieces.

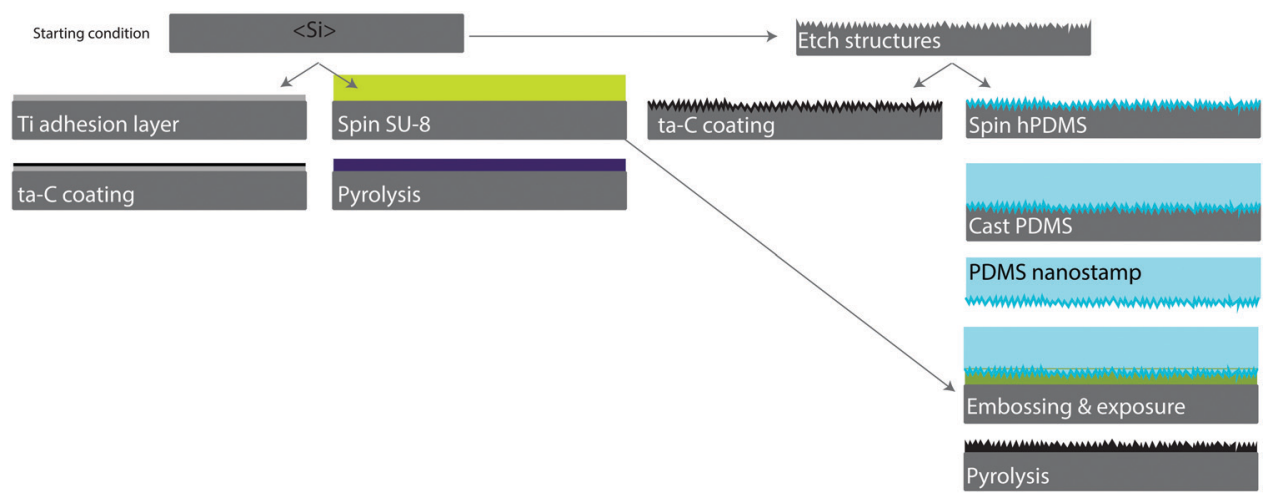

Fig. 1 Fabrication of different electrodes from left to right: ta-C, PyC, nanograss-ta-C and nanograss-PyC. 
PyC was fabricated by pyrolyzing SU-8 photoresist. NanograssPyC samples were fabricated with UV-embossing technique. A PDMS stamp was fabricated with dual-layer process: first, a thin layer of hard-PDMS (hPDMS) was spin coated on the surface of fluoropolymer-coated bSi structured wafer and then normal 10:1 PDMS was casted to provide a bulk structure of the stamp. Prior to spin coating SU-8, the wafers were immersed into BHF (buffered hydrofluoric acid) to remove native oxide from the wafer surface, followed by rinsing in DI-water, spin-drying and moisture removal in an oven at $120{ }^{\circ} \mathrm{C}$ for five minutes. SU-8 50 (MicroChem) was spin coated (9000 rpm, 45 seconds, thickness aim for $13 \mu \mathrm{m}$ ) on the wafers, followed by a ramped soft bake (ramp up $15{ }^{\circ} \mathrm{C} \min ^{-1}$ to $65{ }^{\circ} \mathrm{C}$, hold three minutes, ramp up $15{ }^{\circ} \mathrm{C} \min ^{-1}$ to $95{ }^{\circ} \mathrm{C}$, hold five minutes). The cooling of nanograss sample was stopped at $75{ }^{\circ} \mathrm{C}$ or $65^{\circ} \mathrm{C}$. While the substrate was in $75 / 65^{\circ} \mathrm{C}$, the hPDMSPDMS stamp was placed on top of the soft SU-8 and laminated manually with tweezers to make sure no air bubbles are left between the stamp and the substrate. The sample was then cooled down to room temperature. The solidified polymer film (flat sample) was flood exposed to UV-light for eight seconds, while the substrate-stamp combination (nanograss sample) was flood exposed to UV-light for 16 seconds. UV exposure was followed by a ramped post-exposure bake (PEB) (ramp up $15{ }^{\circ} \mathrm{C} \mathrm{min}{ }^{-1}$ to $95{ }^{\circ} \mathrm{C}$, hold four minutes, cool down $3.75{ }^{\circ} \mathrm{C} \mathrm{min}{ }^{-1}$ to room temperature). The stamps were gently peeled off from the nanograss samples in room temperature, making sure no hPMDS was left on the surface. The samples were diced with Disco DAD3220 dicing saw into $10 \times 10 \mathrm{~mm}^{2}$ pieces and the pyrolysis was carried out in a Nabertherm RS 80/500/11 horizontal tube furnace. The tube was pumped down to pressure of 4 mbar followed by oxygen removal by nitrogen flushing, repeated three times. Low nitrogen flow was left on and the inside pressure was kept at atmospheric pressure during pyrolysis. The furnace was first preheated to $300{ }^{\circ} \mathrm{C}$ for 40 minutes to eliminate the remaining solvent and unreacted monomers from the film. ${ }^{6}$ Then, pyrolysis was carried out at $900{ }^{\circ} \mathrm{C}$ for 60 minutes. The ramp up rates during heating were $200{ }^{\circ} \mathrm{C} \mathrm{h}^{-1}$. Then the furnace was slowly (for 12 hours) cooled down to room temperature. More details of PyC sample fabrication are provided in ref. 15 .

\section{Morphological characterization}

Morphology of the surfaces was characterized by scanning electron microscopy (SEM) as well as atomic force microscopy (AFM). For SEM, Zeiss SUPRA 40 (Oberkochen, Germany) was utilized. For AFM, a Bruker Dimension Icon microscope (Burker, Billerica, MA, USA) in Scan Asyst mode with a Scan Asyst Air tip was used.

\section{Chemical characterization}

Near-edge X-ray absorption fine structure (NEXAFS) data were acquired at a $55^{\circ}$ incidence angle (magic angle) of X-ray incidence using the bending magnet beamline 8-2 at the Stanford Synchrotron Radiation Lightsource (SSRL). Beamline 8-2 is equipped with a spherical grating monochromator, operated using $40 \times 40 \mu \mathrm{m}^{2}$ slits corresponding to a resolution of around $0.2 \mathrm{eV}$. The spot size at the interaction point was around $1 \times 1 \mathrm{~mm}^{2}$, and a flux of $10^{10}$ photons per s was used, at which beam damage is not noticeable even for extended exposure. The X-ray energies for the carbon (C) 1s and oxygen (O) 1s edges were scanned from 260 to $350 \mathrm{eV}$ and 520 to $580 \mathrm{eV}$, respectively. The data were collected both in total electron yield (TEY) and Auger electron yield (AEY) modes using the drain current (amplified by a Keithley picoampmeter) and a cylindrical mirror analyzer (CMA) operated with a pass energy of $200 \mathrm{eV}$ and set to record the main Auger line for the various edges, respectively. The incoming flux was recorded using a nickel grid with a Au sputtered film.

Characterization of the electrode materials was further enhanced by utilizing computational fitting of XAS spectra. Both $\mathrm{C} 1 \mathrm{~s}$ and $\mathrm{O}$ 1s spectra were fitted. The fitting is based on reference spectra that have been computed at density functional theory (DFT) level. The computational spectra are compared with experimental spectrum by optimizing their weighted linear combination, which matches the shape of the experimental spectrum. Unlike in traditional peak fitting, we do not use specific peaks or literature references, but instead we utilize the whole shape of the spectrum within a given energy range and we know exactly where the computational reference spectra are coming from. In addition, we never aim to have a perfect fit since we acknowledge that the selected references cannot totally capture the complexity of experimental reality. Instead, we compare how often the computed spectra occur in the fit. In other words, the weights of the calculated spectra in the fit can be used as semi-quantitative estimates of how much of certain types of differently bonded carbon, or oxygen, is present in the samples. The method has been discussed in depth in ref. 16, 17 and more information about the calculations can be found from $E .^{18-20}$

It is to be noted that the computational references (except pristine graphene, all published in ref. 16) used in the fitting were gained from a-C samples. This may not be an optimal choice as in $\mathrm{sp}^{3}$ rich material most of the characteristic peaks associated with functional groups tend to shift higher in energy compared to corresponding peaks in $\mathrm{sp}^{2}$ rich carbon-based material. ${ }^{17}$ However, as the structure of PyC is not well defined and the fitting code automatically shifts the reference spectra to match the energy alignment of the experimental spectrum that is being fitted, ${ }^{16}$ using the same reference database with ta-C and PyC is well justified. In addition, pristine graphene spectrum was added as a reference to ensure that also features rising from more well organized $\mathrm{sp}^{2}$ bonded carbon, if present, can be captured.

\section{Electrochemistry}

Electron transfer kinetics of two outer sphere redox (OSR) probes, hexaammineruthenium $\mathrm{Ru}\left(\mathrm{NH}_{3}\right)_{6}{ }^{3+}$ and hexachloroiridate $\mathrm{IrCl}_{6}{ }^{2-}$, as well as one inner sphere redox (ISR) probe, DA, were investigated before and after fouling in bovine serum albumin (BSA) or fetal bovine serum (FBS). Cyclic voltammetry was carried out with a Gamry Reference 600 or $600+$ potentiostat in a three-electrode setup with a $\mathrm{Ag} / \mathrm{AgCl}$ reference 
electrode (Radiometer Analytical) and a Pt wire as counter electrode.

The samples were packaged onto copper-clad FR4-PBB sheets and the nanograss-ta-C samples were contacted from the front with a copper tape as bSi forms a native oxide layer, which slows down the electron transport. A circular area with a diameter of $3 \mathrm{~mm}$ was defined with a PTFE tape for all the electrodes.

All the solutions were prepared on the day of the measurement, purged with $\mathrm{N}_{2}$ for at least 15 min and blanketed during experiments. New electrodes were used for each measurement. $1 \mathrm{mM} \mathrm{Ru}\left(\mathrm{NH}_{3}\right)_{6}{ }^{3+}$ in $1 \mathrm{M} \mathrm{KCl}$ and $1 \mathrm{mM} \mathrm{IrCl}_{6}{ }^{2-}$ in $1 \mathrm{M} \mathrm{KCl}$ (pH 7.0) as well as $100 \mu \mathrm{M}$ DA in phosphate buffer solution (PBS, pH 7.4) were investigated. For fouling, samples were immersed in either $2 \%$ bovine serum albumin in PBS or undiluted FBS for 30 minutes at $37{ }^{\circ} \mathrm{C}$ and rinsed in PBS prior to measurements. All measurements were done at room temperature.

In voltammograms, the area increase due to the surface roughness is not taken into account and the area presents only the geometric surface area.

\section{Results and discussion}

\section{Morphology of the surfaces}

Understanding fouling requires careful characterization of topography, structure and surface chemistry. Fig. 2 and 3 present the surface morphologies of the samples, as imaged with SEM and AFM respectively. Both ta-C and PyC thin films are extremely smooth, with root mean square surface roughness (RMS) of $1.2 \mathrm{~nm}$ and $570 \mathrm{pm}$, respectively. The nanograss-ta-C (Fig. 2B) has very sharp and high aspect ratio features and AFM was unable to measure the surface reliably due to convolution effect.

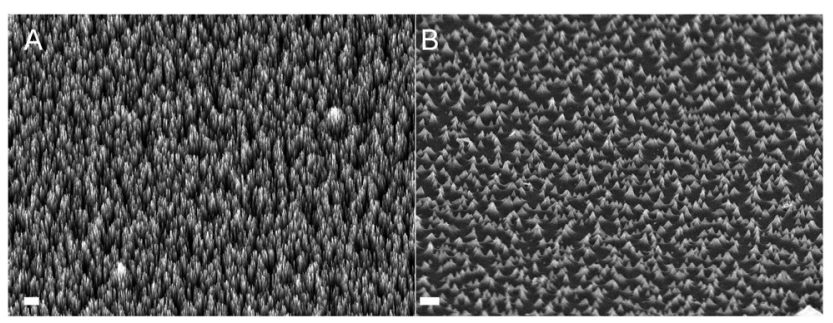

Fig. 2 Scanning electron microscopy image of (A) nanograss-ta-C and (B) nanograss-PyC. scale bar (bottom left) is $2 \mu \mathrm{m}$.
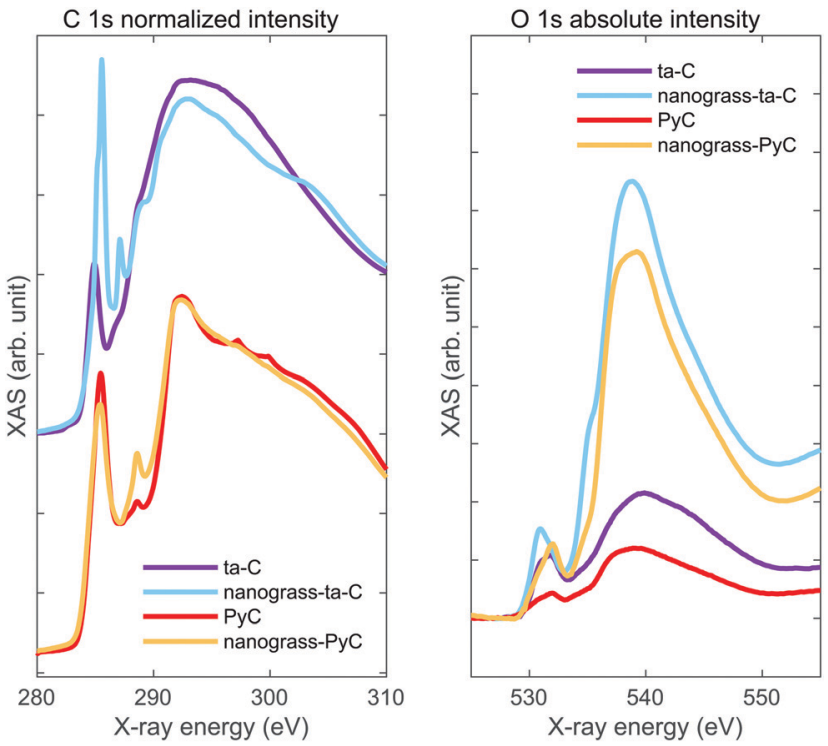

Fig. 4 Near-edge $X$-ray absorption fine structure of carbon 1 s and oxygen 1s edges of ta-C and PyC with and without the nanograss structure.

As the nanograss-PyC is embossed SU-8 resist, which is subsequently pyrolysed, the features are not as sharp as on nanograss-ta-C. RMS of the nanograss-PyC was $170 \mathrm{~nm}$.

\section{Chemical composition of the surfaces}

Fig. 4 presents the $\mathrm{C} 1$ s and $\mathrm{O} 1$ s edges of ta-C and PyC with and without the nanograss structure. Fig. 5 shows the $\mathrm{C} 1 \mathrm{~s}$ and $\mathrm{O} 1 \mathrm{~s}$ edges of the thin film ta-C and $\mathrm{PyC}$ with a computational fit. $\mathrm{N}$ 1s spectra were also acquired but no nitrogen/very small amount of nitrogen was detected for all samples.

To obtain the results shown in Fig. 5, the same structural references were used for computational fitting of ta-C and PyC. The computation fit shows that the biggest difference between ta-C and PyC samples is that despite sharp $\mathrm{sp}^{2} \pi^{*}$ features around $285 \mathrm{eV}$ in the ta-C sample pristine graphene reference is not strongly present, whereas with the PyC sample this reference makes more than $20 \%$ of the fit. This is evident based on to the second sharp $\mathrm{sp}^{2}$ related peak, which indicate that there is some order in the material. ${ }^{21}$ Carbon produced by pyrolysis can result in either graphitizing and non-graphitizing carbons. ${ }^{22}$ The absence of a sharp exciton peak at $291.7 \mathrm{eV}$ in the experimental
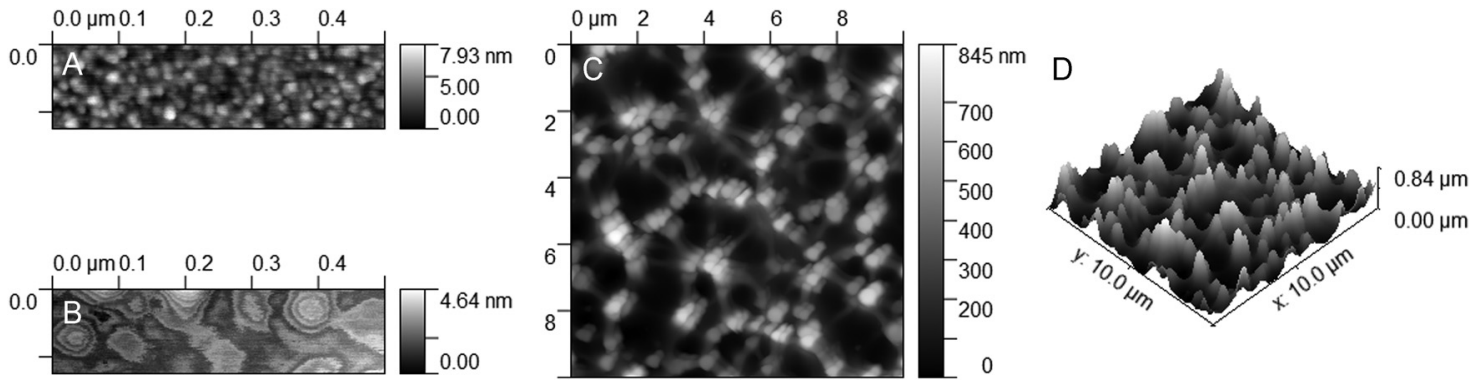

Fig. 3 Atomic force microscopy image of (A) ta-C, (B) PyC and (C and D) nanograss-PyC. 
C 1s normalized intensity

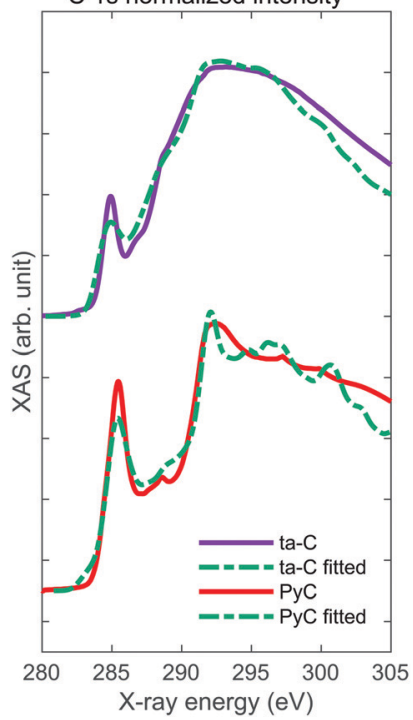

O 1s absolute intensity

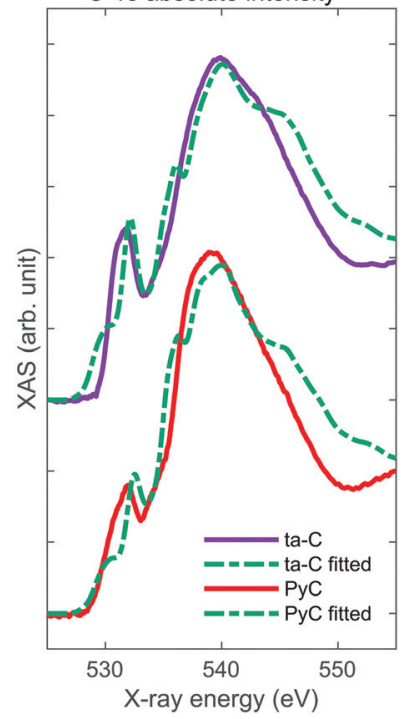

Fig. 5 Near-edge $X$-ray absorption fine structure of carbon 1 s and oxygen 1 s edges of ta-C and PyC with a computational fit.

spectra indicates that PyC examined here is most probably nongraphitizing carbon.

The $\mathrm{C} 1 \mathrm{~s}$ results also suggest that, as expected, ta-C contains a lot more $\mathrm{sp}^{3}$ bonded carbon than PyC. PyC is $\mathrm{sp}^{2}$ rich and possess stronger features related to graphene, as shown by computational fitting (Fig. 5). However, the PyC sample does have some $\mathrm{sp}^{3}$ bonded carbon in it and the results also indicate that there are some highly reactive sp sites present as well. The ta-C sample has more carbon sites that are bonded to oxygen than the PyC sample. Further analysis of O 1s spectra is needed to specify more clearly what kind of oxygen containing groups are present. Fitting results from the $\mathrm{O} 1 \mathrm{~s}$ spectra suggest that the PyC sample has approximately equal amounts of ketone, ether/epoxide and hydroxyl groups, whereas the ta-C sample has slightly more oxygen bonded in the form of ketone than other groups. The computational results also show that there is some carboxylic acid present in the ta-C sample even though it is not clear from the $\mathrm{C} 1 \mathrm{~s}$ spectrum. In case of PyC, carboxylic acid cancels out in both cases. The differences between results gained from $\mathrm{C} 1 \mathrm{~s}$ and $\mathrm{O} 1 \mathrm{~s}$ spectra analysis arise from the fact that many of the features in the spectra are overlapping and some that are hidden in one can be detected from the other. Overall, ta-C appears to contain a significant amount of carbonyl functionalities (ketone $41 \%$ and carboxylic acid $7 \%$ ). In the end, it is to be noted that based our previous results using X-ray photoelectron spectroscopy (XPS), ta-C contains significantly more oxygen functionalities $(8.9 \pm 0.1 \%)$ than PyC $(1.5 \pm 0.1 \%) .{ }^{23}$

The NEXAFS spectrum clearly shows that the surface chemistry of the nanograss surfaces differs from their flat counterparts. Since no existing structural reference gives a reliable computational fit and comparison of nanograss surfaces to their flat counterparts must be done based on experimental spectra alone. While the $\mathrm{C} 1 \mathrm{~s}$ ta- $\mathrm{C}$ spectrum revealed significant amount of $\mathrm{sp}^{3}$ bonded carbon, the nanograss-ta-C carbon is mainly $\mathrm{sp}^{2}$ bonded as indicated by the enhancement of characteristic $\pi^{*}$ peak (ca. $285 \mathrm{eV}$ in the figure) and reduction of the $\sigma^{*}$ characteristics (ca. $291 \mathrm{eV}$ in the figure). Consequently, the carbon on top of bSi surface is in fact amorphous carbon with low $\mathrm{sp}^{3}$ content instead of tetrahedral amorphous carbon. Because of the higher surface area, the carbon layer is thinner on the nanograss surface compared to the flat counterpart. The surface of the ta-C ( $\sim$ nm thickness) is always $\mathrm{sp}^{2}$ carbon. Consequently, with a very thin layer, a high $\mathrm{sp}^{2}$ overall fraction would be expected and the change in the $\mathrm{sp}^{2} / \mathrm{sp}^{3}$ composition might simply be related to the larger surface/bulk ratio in the nanograss structures relative to the flat substrate. However, the ta-C growth process is also expected to be different for the nanograss surface as the carbon-ions come in a glancing angle to the structured surface. Higher glancing angle affects the energy of the impacting carbon-ion, which further affects the formation of $\mathrm{sp}^{3}$ bonds, the thickness of $\mathrm{sp}^{2}$ rich surface layer, the amount of reactive sp carbon on the surface as well as the roughness of the surface. ${ }^{24,25}$ As the oxygen spectrum shows, the nanograss surfaces possess a significantly higher amount of oxygen functionalities than their flat counterparts do. In addition to the actual increase in the number of functional groups, the larger amount of oxygen in these samples is likely to arise from: (i) the dissolution of some of the oxygen into amorphous carbon films and (ii) the silicon oxide layer present on top of the bSi surface as it is highly ambiguous how uniformly the $7 \mathrm{~nm}$ carbon film is actually covering the bSi nanograss substrate. The nanograss-ta-C C 1s spectrum reveals distinctly sharp features, and a particularly sharp peak at $287 \mathrm{eV}$. This indicates that the oxygen-functionalities are oriented, and their local geometry and/or chemistry is similar.

Similarly on nanograss-PyC, the amount of oxygen functionalities is clearly increased compared to flat PyC. As the nanograss-PyC is fabricated with embossing technique, and the thickness of the PyC layer is approximately 2-5 $\mu \mathrm{m}$, their spectrum originates truly from the nanograss-PyC, and not from the underlying silicon wafer. In the nanograss-PyC $\mathrm{C} 1 \mathrm{~s}$ spectrum, the peak at $288.5 \mathrm{eV}$ is significantly higher and sharper compared to flat PyC. This indicates increase in the number of carboxyl functionalities.

To make detailed conclusions about the differences of chemical functionalities and distribution of elements between nanograss surfaces and their flat counterparts, more investigations using, for example, time-of-flight elastic recoil detection analysis (ToF-ERDA) are required. Still, the NEXAFS spectrum revealed striking differences in chemical functionalities of the nanograss surfaces compared to their flat counterparts. Too often, when surfaces are structured, the changes in surface chemistry are neglected and not even characterized. This can lead to false conclusions on the effect of the surface topography.

\section{Electrochemistry and biofouling}

Both hexaammineruthenium $\left(\mathrm{Ru}\left(\mathrm{NH}_{3}\right)_{6}{ }^{3+}\right)$ and hexachloroiridate $\left(\mathrm{IrCl}_{6}{ }^{2-}\right)$ are OSR redox probes, the first carrying a positive charge and the latter a negative charge. DA was tested as an ISR probe. In this study, $\Delta E_{\mathrm{p}}$ values were used as indicators of the 
Table $1 \Delta E_{\mathrm{p}}$ of different redox species measured before and after fouling in BSA or FBS at $50 \mathrm{mV} \mathrm{s}^{-1}$. Percentual increase of the $\Delta E_{\mathrm{p}}$ after fouling in BSA or FBS in parenthesis. Shown error is a standard deviation of at least two samples

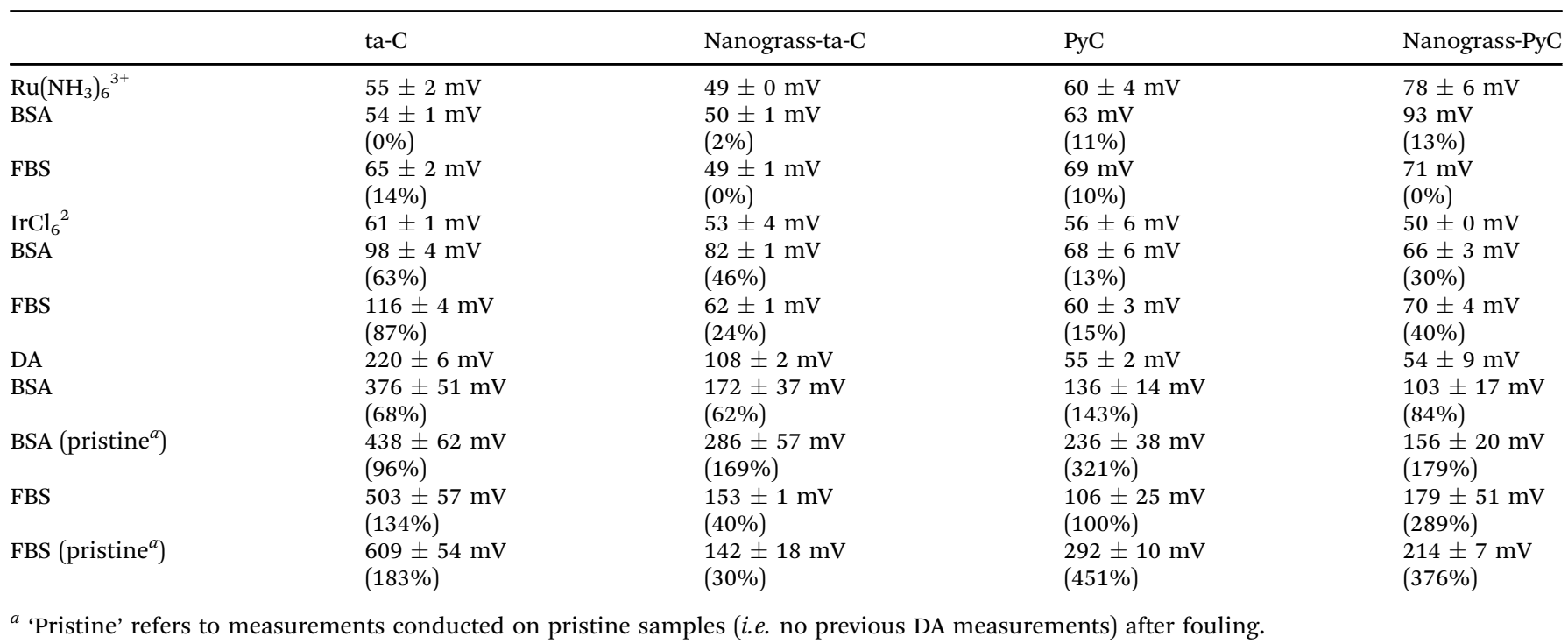

kinetics of the electron transfer reaction. Table 1 summarizes the electron transfer kinetics of the measured probes. We also calculated the $E_{1 / 2}$ values, as a shift in them can be related to protein adsorption. However, with OSR probes, no statistically significant changes were observed in the $E_{1 / 2}$ values after fouling. With DA measurements, the $E_{1 / 2}$ value (Table S1, ESI $\dagger$ ) are in accordance with the changes observed in $\Delta E_{\mathrm{p}}$ after fouling.

\section{Outer sphere probes}

$\mathrm{Ru}\left(\mathrm{NH}_{3}\right)_{6}{ }^{3+}$ shows nearly ideal electron transfer kinetics on ta-C $\left(\Delta E_{\mathrm{p}}=54 \mathrm{mV}\right)$, nanograss-ta-C $\left(\Delta E_{\mathrm{p}}=49 \mathrm{mV}\right)$ and $\mathrm{PyC}$ $\left(\Delta E_{\mathrm{p}}=60\right)$, whereas on nanograss-PyC, the electron transfer kinetics are slightly slower $\left(\Delta E_{\mathrm{p}}=78 \mathrm{mV}\right)$. Voltammograms of $\mathrm{Ru}\left(\mathrm{NH}_{3}\right)_{6}{ }^{3+}$ are presented in Fig. 6 .

BSA fouling has barely any effect on $\mathrm{Ru}\left(\mathrm{NH}_{3}\right)_{6}{ }^{3+}$ kinetics on any of the tested materials. BSA ( $\mathrm{pI}=4.9)$ is adsorbed on the surface, but due to its' anionic charge it does not cause any electrostatic hindrance for cations. BSA has four known conformers: the $\mathrm{N}$ form (dominant in the $\mathrm{pH}$ range of 4.5-8.0), the $\mathrm{F}$ form (at $\mathrm{pH} 4.5-4.0$ ), the most extended $\mathrm{E}$ form (at $\mathrm{pH}$ below $\mathrm{pH} 4$ ), and the $\mathrm{B}$ form (at $\mathrm{pH}$ values above 8 ). ${ }^{26,27}$ Previous research has shown that at neutral $\mathrm{pH}$, when both the BSA and surface are negatively charged, proteins associate with the surface via hydrophobic interactions with no preferential orientation across the surface. ${ }^{28}$ However, there are good evidence for structural changes in BSA upon adsorption. ${ }^{29-31}$

Albumin is the major component also in FBS, but the composition of FBS is more complex containing globulins and fibrinogen as other major components. In addition, regulatory proteins and clotting factors each compose $<1 \%$ of the FBS volume. Although, albumin concentration in plasma is significantly higher than fibrinogen, the adsorbed surface concentration of albumin and fibrinogen are characteristically approximately the same. ${ }^{32}$ Fibrinogen has pI of 5.5, whereas immunoglobulin G, representing
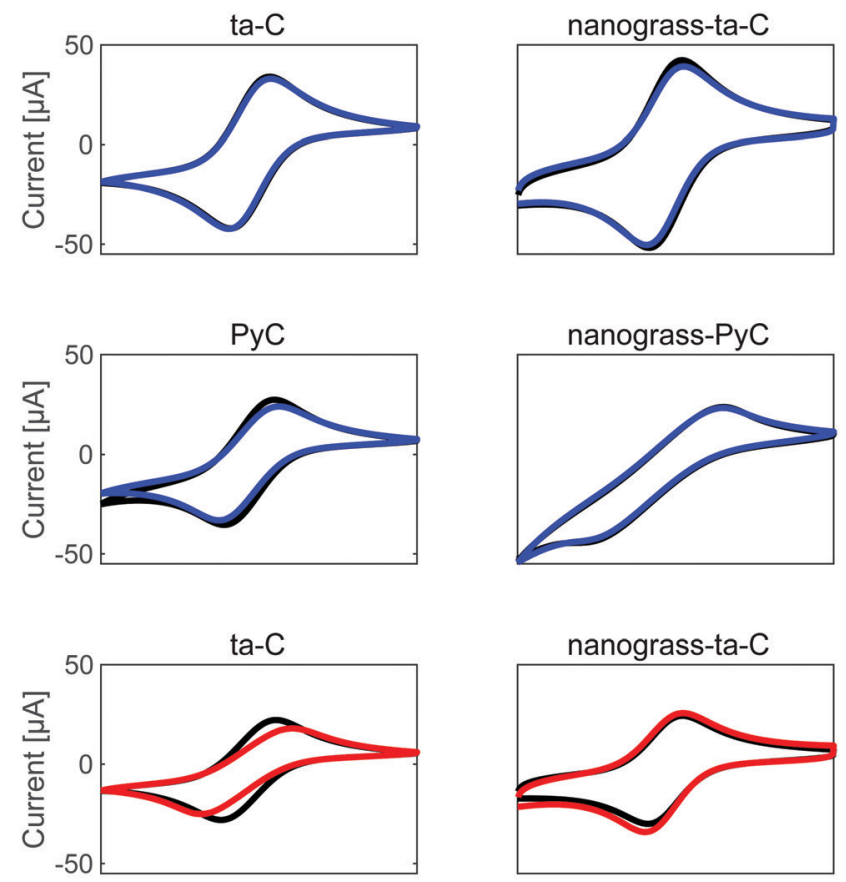

nanograss-ta-C
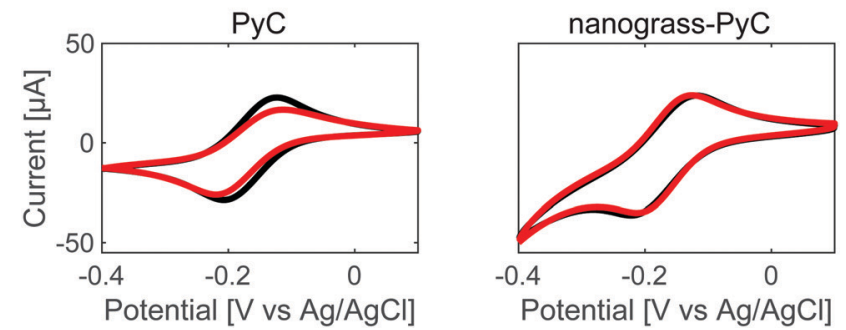

Fig. 6 Voltammograms of $\mathrm{Ru}\left(\mathrm{NH}_{3}\right)_{6}{ }^{3+}\left(400 \mathrm{mV} \mathrm{s}{ }^{-1}\right)$ before (black) and after fouling in BSA (blue) or FBS (red). 
approximately $75 \%$ of serum antibodies, has a pI of $6.6-10.0 .^{33}$ Consequently, IgG can cause electrostatic hindrance to cations. On the other hand, the complex composition of FBS enables better coverage of the surface than a single-protein model of BSA. For example, previous research shows that significant amounts of fibrinogen are able to adsorb on a surface that is already nearly saturated with albumin. ${ }^{34}$ Fibrinogen is also known to undergo major structural changes upon adsorption. For example, on a hydrophobic surface, fibrinogen spreads from an average initial footprint of $100 \mathrm{~nm}^{2}$ to a final footprint near $500 \mathrm{~nm}^{2}$ per molecule while the changes in hydrophilic surface was from 100 to $160 \mathrm{~nm}^{2} .^{35}$ Consequently, the effect of FBS fouling on $\mathrm{Ru}\left(\mathrm{NH}_{3}\right)_{6}{ }^{3+}$ kinetics is more evident than the effect of BSA.

A previous study showed that nanostructured surfaces can lead to higher degrees of surface coverage of proteins per unit area due to lower steric hindrance between particles. ${ }^{36}$ However, with bSi based structures, an increased level of protein adsorption has been observed to occur at the top of the nanopillars, leaving the interpillar space less occupied by proteins. $^{37}$ This could explain, why the effect of FBS fouling on $\mathrm{Ru}\left(\mathrm{NH}_{3}\right)_{6}{ }^{3+}$ kinetics is more pronounced on the flat counterparts. The bSi structure underneath the $7 \mathrm{~nm}$ ta-C coating is a needle-shaped with needle height above $10 \mu \mathrm{m}$ and diameter less than $1 \mu \mathrm{m}$. The diameter of the features is in a larger scale (some 100s $\mathrm{nms}$ ) compared to proteins $(\sim 10 \mathrm{~nm})$. Due to the embossing process, nanograss-PyC features are smoother compared to nanograss-ta-C. However, flat surfaces may promote conformational changes of the proteins that result in increased surface coverage. Distribution of the proteins to the top of the nanopillars and reduced conformational changes could explain, why FBS fouling on nanograss surfaces had negligible effect on $\mathrm{Ru}\left(\mathrm{NH}_{3}\right)_{6}{ }^{3+}$.

$\mathrm{IrCl}_{6}{ }^{2-}$ (Fig. 7) shows nearly ideal electron transfer kinetics on PyC $\left(\Delta E_{\mathrm{p}} \sim 56 \mathrm{mV}\right)$ and on both nanograss surfaces (53 and $50 \mathrm{mV})$. Electron kinetics are slightly slower on ta-C $\left(\Delta E_{\mathrm{p}}\right.$ $60 \mathrm{mV}$ ). For a pure outer sphere electron transfer under diffusion control, $\Delta E_{\mathrm{p}}$ of $57 \mathrm{mV}$ indicates a reversible electron transfer system. Here, the $\Delta E_{\mathrm{p}}$ is less than $57 \mathrm{mV}$ with some of the materials, indicating that adsorption of the probe is involved in the process and the reaction is not a pure OSR reaction.

In contrast to $\mathrm{Ru}\left(\mathrm{NH}_{3}\right)_{6}{ }^{3+}$ kinetics, $\mathrm{IrCl}_{6}{ }^{2-}$ kinetics is heavily affected by BSA fouling on all of the tested materials and the effect of FBS is even more prominent. The electrostatic repulsion between the anionically-charged $\mathrm{BSA}(\mathrm{pI}=4.9)$ adsorbed on the electrode surface and the $\mathrm{IrCl}_{6}{ }^{2-}$ redox species is a probable explanation for the observed stronger fouling effect compared to the effect observed with $\mathrm{Ru}\left(\mathrm{NH}_{3}\right)_{6}{ }^{3+}$. Moreover, the electrostatic interaction between the surface charge of the electrode (highly positive at the formal potential of $\mathrm{IrCl}_{6}{ }^{2-}$ ) and the protein may result in stronger interaction of the protein and the surface and enhanced spreading of the proteins.

Most significant fouling effect on $\mathrm{IrCl}_{6}{ }^{2-}$ kinetics is observed somewhat surprisingly on ta-C, which has often been claimed to possess antifouling properties. "Antifouling”' may refer to several different properties, such as resistance to protein adsorption, resistance to platelet adhesion, or preservation of device
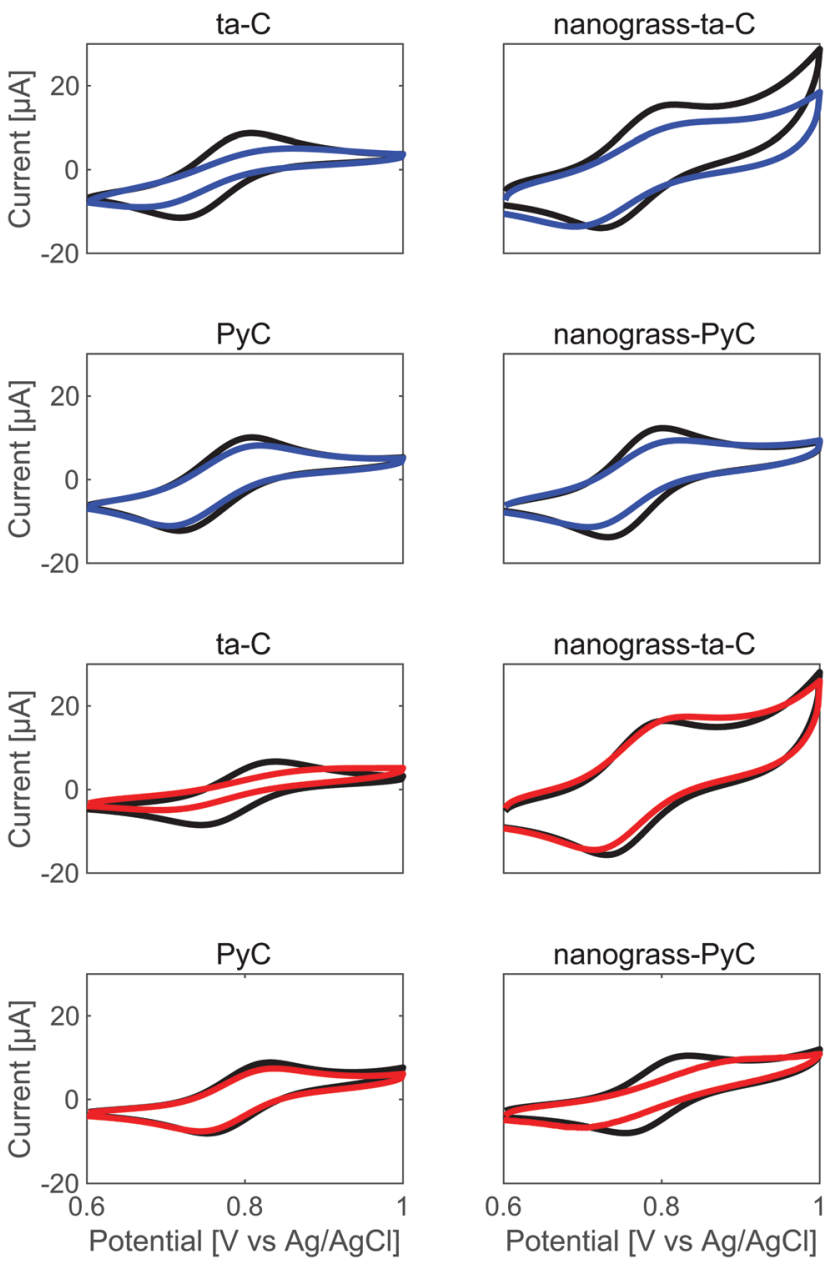

Fig. 7 Voltammograms of $\mathrm{IrCl}_{6}{ }^{2-}\left(400 \mathrm{mV} \mathrm{s}^{-1}\right)$ before (black) and after fouling in BSA (blue) or FBS (red)

function despite of fouling. For example, previous studies have referred diamond-like carbon to exhibit an antifouling ability in solutions containing BSA and human serum compared to glassy carbon. ${ }^{38}$ The authors showed that $\mathrm{Fe}(\mathrm{CN})_{6}{ }^{4-/ 3-}$ signal was unaffected by fouling on diamond-like carbon (DLC). On the other hand, ta-C is known to bind BSA more strongly than fibrinogen. ${ }^{39,40}$ Moreover, it has been proposed that platelet adhesion is inversely proportional to the albumin to fibrinogen ratio: the higher the ratio is the lower the number of adhering platelets. ${ }^{41}$ For comparison, the albumin to fibrinogen ratio is 1.24 for DLC and 0.76 for silicone elastomer. ${ }^{42}$ DLC might not resist protein adsorption, but it has antifouling properties towards platelet adhesion and preservation of OSR probe signal.

Other studies clearly support the idea that conformation of fibrinogen is a critical determinant of platelet adhesion rather than the amount of fibrinogen adsorbed. ${ }^{43}$ Indeed, the amount of adsorbed fibrinogen has been found to be relatively high on PyC, while the platelet retention was lower compared to titanium and polystyrene. ${ }^{40}$ The authors concluded that PyC causes conformational changes to fibrinogen that contribute to the enhanced hemocompatibility. Similarly, both the amount 
of adsorbed proteins as well as their conformation are relevant for the device function.

Electron transfer kinetics of $\mathrm{IrCl}_{6}{ }^{2-}$ is significantly more affected on ta-C $\left(\Delta E_{\mathrm{p}}\right.$ increase $63-84 \%$ after fouling) compared to other tested materials ( $\Delta E_{\mathrm{p}}$ increase $13-46 \%$ after fouling). The stronger presence of carbonyl functional groups (shown by the NEXAFS) on ta-C makes the surface less hydrophobic, or rather a "hydroneutral" surface, compared to PyC. Moreover, the structure of nanograss surfaces further increases their hydrophobic nature. As previously discussed, the hydrophilic/ hydrophobic nature of the surface affects the conformation of proteins. The spreading rate of both albumin and fibrinogen has been observed to increase with substrate hydrophobicity, resulting in a larger footprint per molecule and decreased overall coverage. ${ }^{34}$ This lower coverage and higher degree of denaturation could, at least partially, explain the decreased effect of fouling on $\mathrm{IrCl}_{6}{ }^{2-}$ kinetics observed on PyC and nanograss surfaces compared to ta-C. This is based on the fact that the way to affect the kinetics of OSR probes is to push them further away from the electrode surface and thus affect the adiabaticity of the electron transfer reactions. It may be that in the case of more hydrophobic PyC electrodes the more denatured proteins form a thinner (albeit more compact) layer that does not increase the electron transfer distance as much as in the case of ta-C.

Differences in the effect of fouling on OSR probe electron transfer kinetics is, at least, partially due to the electrostatic repulsion or attraction of the probe and negatively charged proteins. Likewise, the formal oxidation potentials may also affect the results: $\mathrm{Ru}\left(\mathrm{NH}_{3}\right)_{6}{ }^{3+}$ is oxidized at $c a .-100 \mathrm{mV}$ vs. $\mathrm{Ag} / \mathrm{AgCl}$, while $\mathrm{IrCl}_{6}{ }^{2}$ oxidizes at ca. $800 \mathrm{mV}$ vs. $\mathrm{Ag} / \mathrm{AgCl}$. Consequently, in the $\mathrm{Ru}\left(\mathrm{NH}_{3}\right)_{6}{ }^{3+}$ measurement, the surfaces carry a negative charge whereas surface charge is positive during the $\mathrm{IrCl}_{6}{ }^{2-}$ measurements. However, a previous paper has shown decreasing rate of albumin adsorption with increasing surface charge of glassy carbon (calculated charges from $-2 \mu \mathrm{C} \mathrm{cm}^{-2}$ to $8.1 \mu \mathrm{C} \mathrm{cm}^{-2}$, while shifting potential from $-80 \mathrm{mV}$ to $550 \mathrm{mV} v s$. $\mathrm{Ag} / \mathrm{AgCl}) .{ }^{44}$ Here, the fouling occurred on non-charged environment and the effect of BSA and FBS fouling on OSR probe electron transfer kinetics were more pronounced at $800 \mathrm{mV}$ vs. $\mathrm{Ag} / \mathrm{AgCl}$ than at $-100 \mathrm{mV} v s$. Ag/AgCl. Here, however, the potential applied during cycling can affect the strength of protein adsorption, and possibly spreading of the protein, due to electrostatic interaction. The effect of surface charge on protein conformation and coverage and the corresponding biofouling effect deserves more attention. However, the combined effect of hydrophobicity and sign of the surface charge appear to point towards a higher fouling effect in the case of ta-C surface.

\section{DA}

DA ( $\mathrm{pI}=8.88)$ is an ISR probe and it undergoes both electrochemical and chemical reactions on electrode materials. Table 1 shows the $\Delta E_{\mathrm{p}}$ prior to fouling and the percentage change of it after fouling. Fig. 8 presents the voltammograms of DA measurements. On PyC, the DA reaction kinetics are rapid as DA adsorbs on PyC. ${ }^{2}$ The adsorption behaviour is most likely explained by the $\pi$-orbital interaction between graphene
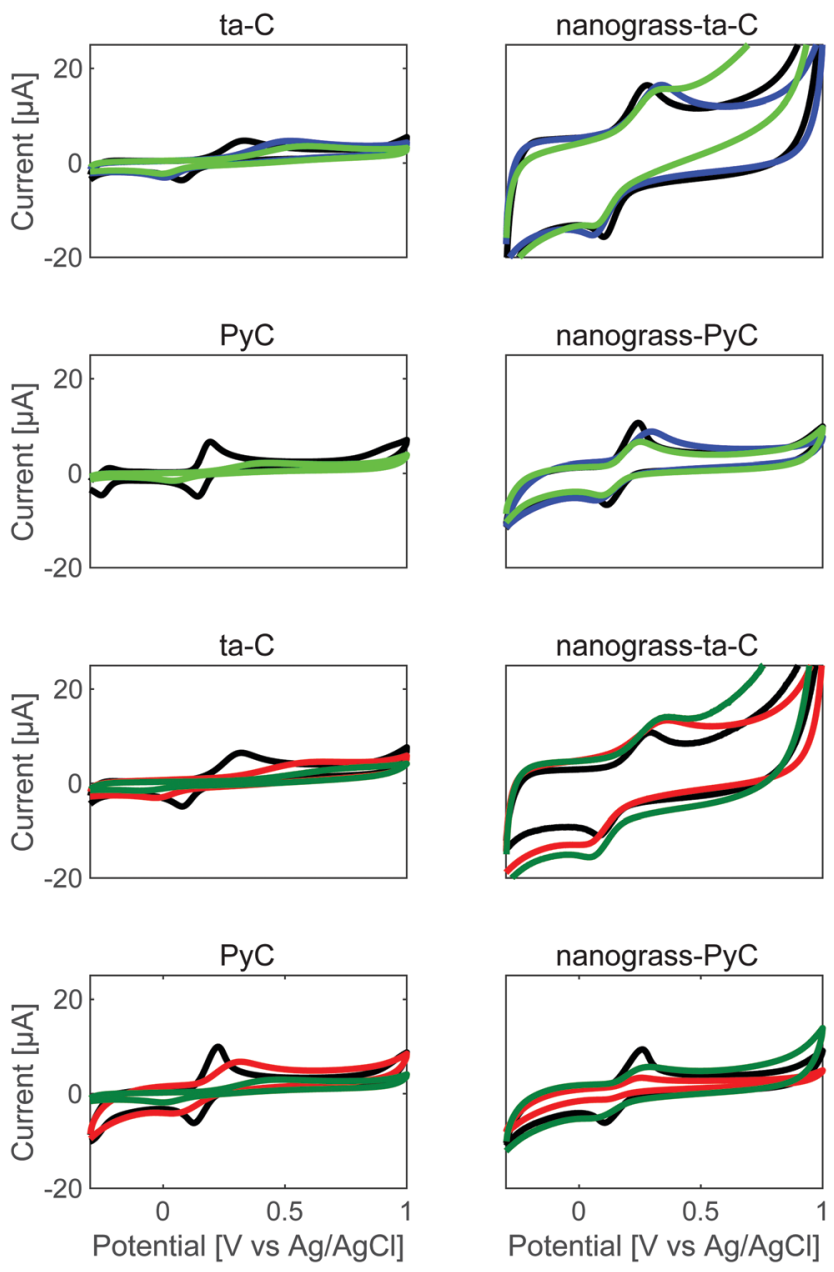

Fig. 8 Voltammograms of DA (400 mV s${ }^{-1}$ ) before (black) and after fouling in BSA (blue) or FBS (red). Separate samples were measured only after BSA (light green) or FBS (dark green) fouling.

hexagons (which is evident based on XAS results) and the benzene ring of DA. ${ }^{2}$ Furthermore, the adsorption of the DA layer on the electrode is known to be catalytic towards DA oxidation and reduction, ${ }^{45}$ partly explaining the rapid electron transfer kinetics. On PyC, DA shows both diffusion and adsorption-defined reaction kinetics. ${ }^{2}$ Moreover, on PyC, the dopaminechrome/leucodopaminechrome (DAC/LDAC) peak pair (around $-100 \mathrm{mV} v s$. $\mathrm{Ag} / \mathrm{AgCl}$ ) is symmetric and strongly shifted to cathodic direction from DA/dopamine-o-quinone (DAQ) peak. This indicates that in addition to DAQ, also LDAC adsorbs on PyC.

On ta-C and nanograss-ta-C the electron transfer kinetics are sluggish compared to both PyC surfaces. Sluggish electron transfer kinetics of other catechols has also been observed on ta-C. ${ }^{46}$

The concentration of DA utilized in this paper $(100 \mu \mathrm{M})$ is relatively high and some DA is possibly left on the surface as is also evident with PBS cycling after the DA fouling. Under a suitable environment $(\mathrm{pH}>7.5$, DA concentrations higher than $2 \mathrm{mg} \mathrm{mL}{ }^{-1}$ ), polydopamine formation occurs spontaneously. ${ }^{47}$ However, electrochemical fouling is typical for DA even at lower 
concentrations, as its oxidation products are very reactive. We have previously shown that the ability to recover the activity of the PyC electrode by cycling in $\mathrm{H}_{2} \mathrm{SO}_{4}$ after fouling with DA is inferior compared to several other carbon materials possibly due to stronger adsorption of DA on the surface. ${ }^{23}$ The stronger fouling of PyC is consistent with the results from OSR probes. ISR probes require adsorption on the electrode surface for the redox reactions can occur. In this case the thinner, but more strongly adhered denatured protein layer on PyC prevents adsorption from occurring and makes the reactions sluggish. Poorer recovery of PyC electrodes after fouling further supports this suggestion.

It is possible that precycling the samples in DA could protect the samples from biofouling to some extent. Samples subjected to biological fouling prior to DA measurements, showed larger change in $\Delta E_{\mathrm{p}}$ (see Table 1) after fouling than the samples that are fouled only after initial DA measurements. An exception for this behavior was the nanograss-ta-C in the case of FBS, where the change in $\Delta E_{\mathrm{p}}$ is smaller on pristine surface.

\section{Conclusions}

The NEXAFS spectrum revealed striking differences in chemical functionalities of the surfaces. PyC contained very small amount of oxygen functionalities (1.5\%) and equal amounts of ketone, hydroxyl and ether/epoxide groups, while ta-C contained significantly more $(8.9 \%)$ of oxygen functionalities, and strong presence of carbonyl groups (41\% of ketone and $7 \%$ of carboxyl functionalities). Moreover, the amount of oxygen functionalities was significantly increased on nanograss surfaces compared to the flat counterparts, and nanograss-ta-C no longer possessed significant amounts of $\mathrm{sp}^{3}$ carbon.

Both tested OSR probes, $\mathrm{Ru}\left(\mathrm{NH}_{3}\right)_{6}{ }^{3+}$ and $\mathrm{IrCl}_{6}{ }^{2-}$, showed rapid electron transfer kinetics on all of the materials prior to fouling. $\mathrm{Ru}\left(\mathrm{NH}_{3}\right)_{6}{ }^{3+}$ electron transfer kinetics were not significantly affected by neither BSA or FBS fouling. In contrast, $\mathrm{IrCl}_{6}{ }^{2-}$ kinetics were clearly affected by both types of fouling on all of the tested materials, possibly mainly because of the electrostatic interaction of the positively charged surface $\left(\mathrm{IrCl}_{6}{ }^{2-}\right.$ oxidation around $800 \mathrm{mV}$ vs. $\mathrm{Ag} / \mathrm{AgCl}$ ) and negatively charged protein.

The stronger presence of carbonyl functional groups (shown by the NEXAFS) on ta-C makes the surface less hydrophobic or rather a "hydroneutral" surface, compared to PyC. Due to the hydrophobic nature of PyC, (i) conformational changes of proteins are more likely and interactions between the surface and protein are stronger. Consequently, (ii) the proteins take a larger footprint per molecule and the overall coverage is decreased. (iii) The effect of fouling on OSR probes is negligible on PyC as electron tunneling is not significantly hindered due to the thinner albeit more strongly adhered protein layer. In contrast, (iv) on ta-C the proteins form a more dense layer (v) affecting the electron tunneling through a thicker layer and slowing down the electron transfer kinetics of OSR probes. DA was tested as an ISR probe and the kinetics is heavily dependent on the material, DA electron transfer kinetics being very fast on PyC and sluggish on ta-C. This is reasonable due to $\pi$-orbital interaction between graphene hexagons (shown by NEXAFS and computational pristine graphene reference making more than $20 \%$ of the fit) of PyC and DA. DA electron transfer kinetics were heavily affected on all surfaces by fouling, $\Delta E_{\mathrm{p}}$ increase being $30-451 \%$ after fouling. The fouling effect on DA appears to be opposite compared to OSR probes. The fouling effect on pristine surface was significantly smaller on ta-C $\left(\Delta E_{\mathrm{p}}\right.$ increase $\left.96-183 \%\right)$ compared to PyC $\left(\Delta E_{\mathrm{p}}\right.$ increase $321-451 \%$ ). On PyC, due the hydrophobic nature of the surface, the spreading of the proteins was increased and the interaction of the proteins is stronger. Consequently, (vi) the access of the probe to the inner sphere was prevented, making the effect of fouling stronger by slowing down the electron transfer kinetics on PyC.

\section{Conflicts of interest}

There are no conflicts to declare.

\section{Acknowledgements}

EP acknowledges funding from Academy of Finland (\#321996 and \#328854) and Jane and Aatos Erkko Foundation. NW acknowledges funding from Business Finland (grant number 2117731), the Orion Research Foundation sr and the Foundation for Aalto University Science and Technology. The authors acknowledge the provision of facilities by Aalto University OtaNanoMicronova Nanofabrication Center, OtaNano-Nanomicroscopy Center (Aalto-NMC) and RawMatters research infrastructure (RAMI). This project has received funding from the European Union's Horizon 2020 research and innovation programme under the Marie Skłodowska-Curie grant agreement no. 841621. SLAC National Accelerator Laboratory, is supported by the U.S. Department of Energy, Office of Science, Office of Basic Energy Sciences under contract no. DE-AC02-76SF00515. The authors wish to acknowledge CSC - IT Center for Science, Finland, for the computational resources provided for this work.

\section{References}

$1 \mathrm{~N}$. Wisniewski and M. Reichert, Methods for reducing biosensor membrane biofouling, Colloids Surf., B, 2000, 18, 197-219.

2 E. Peltola, J. J. Heikkinen, K. Sovanto, S. Sainio, A. Aarva and S. Franssila, et al., SU-8 based pyrolytic carbon for the electrochemical detection of dopamine, J. Mater. Chem. B, 2017, 5, 9033-9044.

3 T. A. Horbett, Chapter 13 Principles underlying the role of adsorbed plasma proteins in blood interactions with foreign materials, Cardiovasc. Pathol., 1993, 2, 137-148.

4 B. L. Hanssen, S. Siraj and D. K. Y. Wong, Recent strategies to minimise fouling in electrochemical detection systems, Rev. Anal. Chem., 2016, 35, 1-28. 
5 D. L. Robinson, A. Hermans, A. T. Seipel and R. M. Wightman, Monitoring Rapid Chemical Communication in the Brain, Chem. Rev., 2008, 108(7), 2554-2584.

6 R. Martinez-Duarte, SU-8 Photolithography as a Toolbox for Carbon MEMS, Micromachines, 2014, 5(3), 766-782.

7 K. Rechendorff, M. B. Hovgaard, M. Foss, V. P. Zhdanov and F. Besenbacher, Enhancement of protein adsorption induced by surface roughness, Langmuir, 2006, 22, 10885-10888.

8 T. D. B. Nguyen-Vu, H. Chen, A. M. Cassell, R. J. Andrews, M. Meyyappan and J. Li, Vertically aligned carbon nanofiber architecture as a multifunctional 3-D neural electrical interface, IEEE Trans. Biomed. Eng., 2007, 56, 1121-1128.

9 G. Hazell, P. W. May, P. Taylor, A. H. Nobbs, C. C. Welch and B. Su, Studies of black silicon and black diamond as materials for antibacterial surfaces, Biomater. Sci., 2018, 6, 1424-1432.

10 P. W. May, M. Clegg, T. A. Silva, H. Zanin, O. Fatibello-Filho and V. Celorrio, et al., Diamond-coated "black silicon" as a promising material for high-surface-area electrochemical electrodes and antibacterial surfaces, J. Mater. Chem. B, 2016, 4, 5737-5746.

11 R. Trouillon and D. O'Hare, Comparison of glassy carbon and boron doped diamond electrodes: resistance to biofouling, Electrochim. Acta, 2010, 55(22), 6586-6595.

12 A. Baszkin and D. J. Lyman, The interaction of plasma proteins with polymers. I. Relationship between polymer surface energy and protein adsorption/desorption, J. Biomed. Mater. Res., 1980, 14(4), 393-403.

13 L. Sainiemi, V. Jokinen, A. Shah, M. Shpak, S. Aura and P. Suvanto, et al., Non-Reflecting Silicon and Polymer Surfaces by Plasma Etching and Replication, Adv. Mater., 2011, 23(1), 122-126.

14 T. Palomäki, N. Wester, M. A. Caro, S. Sainio, V. Protopopova and J. Koskinen, et al., Electron transport determines the electrochemical properties of tetrahedral amorphous carbon (ta-C) thin films, Electrochim. Acta, 2017, 225, 1-10.

15 J. J. Heikkinen, E. Peltola, N. Wester, J. Koskinen, T. Laurila and S. Franssila, et al., Fabrication of Micro- and Nanopillars from Pyrolytic Carbon and Tetrahedral Amorphous Carbon, Micromachines, 2019, 10(8), 510.

16 A. Aarva, V. L. Deringer, S. Sainio, T. Laurila and M. A. Caro, Understanding X-ray Spectroscopy of Carbonaceous Materials by Combining Experiments, Density Functional Theory, and Machine Learning. Part II: Quantitative Fitting of Spectra, Chem. Mater., 2019, 31(22), 9256-9267.

17 A. Aarva, V. L. Deringer, S. Sainio, T. Laurila and M. A. Caro, Understanding X-ray Spectroscopy of Carbonaceous Materials by Combining Experiments, Density Functional Theory, and Machine Learning. Part I: Fingerprint Spectra, Chem. Mater., 2019, 31(22), 9243-9255.

18 J. Enkovaara, C. Rostgaard, J. J. Mortensen, J. Chen, M. Dułak and L. Ferrighi, et al., Electronic structure calculations with GPAW: a real-space implementation of the projector augmented-wave method, J. Phys.: Condens. Matter, 2010, 22(25), 253202.
19 GPAW: DFT and beyond within the projector-augmented wave method-GPAW. [cited 2020 Mar 5]. Available from: https://wiki.fysik.dtu.dk/gpaw/.

20 M. Taillefumier, D. Cabaret, A. M. Flank and F. Mauri, X-ray absorption near-edge structure calculations with the pseudopotentials: Application to the $\mathrm{K}$ edge in diamond and (formula presented)-quartz, Phys. Rev. B: Condens. Matter Mater. Phys., 2002, 66(19), 1-8.

21 S. Sainio, et. al., Trends in carbon, oxygen and nitrogen core spectra in the X-ray Absorption Spectroscopy of carbon nanomaterials - a guide for the perplexed, 2020, submitted.

22 R. E. Franklin, Crystallite growth in graphitizing and nongraphitizing carbons, Proc. R. Soc. London, Ser. A, 1951, 209(1097), 196-218.

23 E. Peltola, S. Sainio, K. B. Holt, T. Palomäki, J. Koskinen and T. Laurila, Electrochemical Fouling of Dopamine and Recovery of Carbon Electrodes, Anal. Chem., 2018, 90, 1408-1416.

24 M. A. Caro, V. L. Deringer, J. Koskinen, T. Laurila and G. Csányi, Growth Mechanism and Origin of High $\mathrm{sp}^{3}$ Content in Tetrahedral Amorphous Carbon, Phys. Rev. Lett., 2018, 120(16), 166101.

25 M. A. Caro, G. Csányi, T. Laurila and V. L. Deringer, Machine-learning-driven simulated deposition of carbon films: from low-density to diamond-like amorphous carbon, arXiv:2006.09760.

26 D. C. Carter and J. X. Ho, Structure of serum albumin, Adv. Protein Chem., 1994, 45(C), 153-176.

27 D. H. Tsai, F. W. Delrio, A. M. Keene, K. M. Tyner, R. I. MacCuspie and T. J. Cho, et al., Adsorption and conformation of serum albumin protein on gold nanoparticles investigated using dimensional measurements and in situ spectroscopic methods, Langmuir, 2011, 27(6), 2464-2477.

28 R. P. Putra, Y. Ikumura, H. Horino, A. Hori and I. I. Rzeznicka, Adsorption and Conformation of Bovine Serum Albumin with Blue-Emitting Gold Nanoclusters at the Air/Water and Lipid/ Water Interfaces, Langmuir, 2019, 35(50), 16576-16582.

29 C. E. Giacomelli and W. Norde, The adsorption-desorption cycle. Reversibility of the BSA-silica system, J. Colloid Interface Sci., 2001, 233(2), 234-240.

30 W. Norde and C. E. Giacomelli, Conformational changes in proteins at interfaces: From solution to the interface, and back, Macromol. Symp., 1999, 145(1), 125-136.

31 W. Norde and C. E. Giacomelli, BSA structural changes during homomolecular exchange between the adsorbed and the dissolved states in J. Biotechnol., Elsevier, 2000, pp. 259-268.

32 T. A. Horbett, Chapter II.1.2 Adsorbed Proteins on Biomaterials ed. B. Ratner, A. Hoffman, F. Schoen and J. Lemons, Biomaterials Science An Introduction to Materials in Medicine, Elsevier, 3rd edn, 2013.

33 Y. Jin, G. Luo, T. Oka and T. Manabe, Estimation of isoelectric points of human plasmaproteins employing capillary isoelectric focusing and peptide isoelectric point markers, Electrophoresis, 2002, 23(19), 3385-3391.

34 C. F. Wertz and M. M. Santore, Effect of surface hydrophobicity on adsorption and relaxation kinetics of albumin and 
fibrinogen: Single-species and competitive behavior, Langmuir, 2001, 17(10), 3006-3016.

35 M. M. Santore and C. F. Wertz, Protein spreading kinetics at liquid-solid interfaces via an adsorption probe method, Langmuir, 2005, 21(22), 10172-10178.

36 B. M. Manzi, M. Werner, E. P. Ivanova, R. J. Crawford and V. A. Baulin, Simulations of Protein Adsorption on Nanostructured Surfaces, Sci. Rep., 2019, 9, 1.

37 D. H. K. Nguyen, V. T. H. Pham, M. Al Kobaisi, C. Bhadra, A. Orlowska and S. Ghanaati, et al., Adsorption of Human Plasma Albumin and Fibronectin onto Nanostructured Black Silicon Surfaces, Langmuir, 2016, 32, 10744-10751.

38 T. Goto, T. Yasukawa, K. Kanda, S. Matsui and F. Mizutani, Inhibition of Electrochemical Fouling against Biomolecules on a Diamond-Like Carbon Electrode, Anal. Sci., 2011, 27(1), 91-94.

39 M. Fedel, A. Motta, D. Maniglio and C. Migliaresi, Surface properties and blood compatibility of commercially available diamond-like carbon coatings for cardiovascular devices, J. Biomed. Mater. Res., Part B, 2008, 90B(1), 338-349.

40 S. Forti, L. Lunelli, C. D. Volpe, S. Siboni, L. Pasquardini and A. Lui, Hemocompatibility of pyrolytic carbon in comparison with other biomaterials, Diamond Relat. Mater., 2011, 20(5-6), 762-769.
41 I. Dion, X. Roques, H. Baquey, E. Baudet, B. Basse Cathalinat and N. More, Hemocompatibility of Diamond-Like Carbon Coating, Biomed. Mater. Eng., 1993, 3(1), 51-55.

42 F. Cui and D. Li, A review of investigations on biocompatibility of diamond-like carbon and carbon nitride films, Surf. Coat. Technol., 2000, 131(1-3), 481-487.

43 T. A. Horbett, Fibrinogen adsorption to biomaterials, J. Biomed. Mater. Res., Part A, 2018, 106(10), 2777-2788.

44 P. Bernabeu and A. Caprani, Influence of surface charge on adsorption of fibrinogen and/or albumin on a rotating disc electrode of platinum and carbon, Biomaterials, 1990, 11(4), 258-264.

45 S. H. Duvall and R. L. McCreery, Self-catalysis by catechols and quinones during heterogeneous electron transfer at carbon electrodes, J. Am. Chem. Soc., 2000, 122(28), 6759-6764.

46 T. Palomäki, S. Chumillas, S. Sainio, V. Protopopova, M. Kauppila and J. Koskinen, et al., Electrochemical reactions of catechol, methylcatechol and dopamine at tetrahedral amorphous carbon (ta-C) thin film electrodes, Diamond Relat. Mater., 2015, 59, 30-39.

47 Y. Liu, K. Ai and L. Lu, Polydopamine and its derivative materials: Synthesis and promising applications in energy, environmental, and biomedical fields, Chem. Rev., 2014, 114(9), 5057-5115. 